\title{
Soziale Arbeit als Agens der Politik
}

\author{
Über Sozialpolitik und über Sozialpolitik hinaus
}

\author{
Albert Mühlum
}

Soziale Arbeit hat es unvermeidlich mit Verlustangst und Verlusterfahrung zu tun. Sie muss deshalb sensibel sein für die Sinnfrage und für spirituelle Anliegen, forderte Albert Mühlum in seinem Vortrag auf der letzten Jahrestagung der Deutschen Gesellschaft für Soziale Arbeit.

Die Soziale Arbeit ist bisher mehr Agent als Agens der Politik, mehr Vertreter als treibende Kraft, obwohl die Politik der sozialprofessionellen Expertise doch dringend bedarf. Dabei geht es sowohl um die Wahrnehmung und Bearbeitung Sozialer Probleme als auch um Schwierigkeiten und Fallstricke des Sozialstaats, die bewusst sein müssen, um gegensteuern zu können. Dabei reichen die staatlichen Steuerungsmodi Gesetz und Geld nicht aus. In den Grenzsituationen des Lebens, mit denen es die Soziale Arbeit oft zu tun hat, ist Spiritualität eine besondere Ressource - jenseits aller (Sozial-)Politik. Insofern müssen Sozialarbeitsforschung, Sozialarbeitsphilosophie und Professionalität mit der Sozialpolitik zusammenwirken, um Sozialleistungen optimieren und den künftigen Gesamthilfebedarf abdecken zu können - auf dem Weg zu einer sozial integrierten Gesellschaft.

Soziale Arbeit als Agens der Politik? Wer hätte da nicht Zweifel. Treibende Kraft sind gewöhnlich andere, vorzugweise ökonomische Interessen. $\mathrm{Ob}$ eine Re-Politisierung der Sozialarbeit das ändern könnte, ist eine offene und durchaus strittige Frage (Lallinger/Rieger 2007). Vermutlich steht die sozialpolitische - be-

Prof. Dr. Albert Mühlum war bis zu seiner Pensionierung Hochschullehrer für Sozialpolitik und Sozialarbeitswissenschaft an der Fachhochschule Heidelberg und Lehrbeauftragter der Universität Heidelberg. Er war lange Jahre Vorstandsmitglied der Deutschen Gesellschaft für Soziale Arbeit e. V. E-Mail a.muehlum@t-online.de stimmt aber die sozialwissenschaftliche Kompetenz der Lehrenden und Forschenden im Bereich Sozialer Arbeit im umgekehrten Verhältnis zu ihrer sozialpolitischen Potenz. Bei Politikern dürfte das Gegenteil zutreffen. Was also läge näher, als die Expertise der Sozialen Arbeit und ihrer hundertjährigen Berufsgeschichte (Wendt 2008) für die Gestaltung des Sozialen zu nutzen - mit der Sozialpolitik und über die Sozialpolitik hinaus? Dazu werden im Folgenden - fragmentarisch vier Aspekte angesprochen: die Wahrnehmung sozialer Probleme, Schwierigkeiten der Sozialpolitik, Fallstricke des Sozialstaats und Spiritualität als Ressource.

\section{Wahrnehmung Sozialer Probleme}

Schon der Blick auf soziale Anliegen trifft auf Widerstände. Von Sozialarbeit sprechen heißt ja, sich mit Problemen und Not zu beschäftigen. Und wer befasst sich schon gern mit den Schattenseiten des Lebens? Von Königin Victoria wird berichtet, wie sie einst beim Ausritt ungewollt die Slums von London streifte und - erschrocken über das Elend - ihren Fächer vor die Augen hielt. Der Fächer der Königin als Symbol für Verdrängung. Eine andere Königin, Marie-Antoinette, soll am Vorabend der Revolution auf die Klage, das Volk habe kein Brot mehr, geantwortet haben: "Sollen sie doch Kuchen essen. «

Nun sind unsere Politiker weder königlich noch völlig weltfremd - oder nimmt der Fächer heute nur andere Formen an? Eine erste Aufgabe besteht darin, den Blick auf soziale Probleme freizulegen und genauer hinzuschauen - auf Armut, Ausgrenzung, Benachteiligung. In dieser Zeitschrift muss weder mit Fallstudien noch mit Zahlen erläutert werden, was dies für Teilhabegerechtigkeit und Entwicklungschancen bedeutet. Die dramatischen »Abstiegsprozesse in den Zentren der Sozialstaaten « (Drilling 2004) sind den in der Sozialarbeit Tätigen bekannt, scheinen aber Politiker und Öffentlichkeit immer wieder zu überraschen. Wenn die Soziale Arbeit den Fächer beiseite schiebt, auf das Ausmaß und die Dynamik der Armutsprozesse aufmerksam macht und aus intimer Kenntnis der Nöte diese skandalisiert macht sie sich unbeliebt, wird zum personifizierten schlechten Gewissen der Gesellschaft. Aber da sie Probleme nicht nur aufdeckt, sondern löst, trägt sie zur Entlastung bei, fördert Systemloyalität und wird so paradoxerweise auch zum guten Gewissen des Sozialstaats. Diese Ambivalenz teilt sie mit der Sozialpolitik, deren doppelte Funktion im Kapitalismus schon Eduard Heimann beschrieb - systemverändernd und systemerhaltend zugleich.

Wie alle komplexen Systeme müssen auch soziale Systeme Hilfe organisieren, wenn Grundanliegen bedroht sind, das wurde schon in den 1980er Jahren als »ökosoziales Paradigma der Sozialarbeit « nutzbar gemacht (Mühlum/Olschowy/ Oppl/Wendt 1986). In der Krise der Moderne (Amitai Etzioni nennt sie eine schwere soziale Erkrankung) wird das Gelingen der Lebensentwürfe schwieriger und die Gerechtigkeitslücke wird größer: zwischen den Generationen und den Geschlechtern, Rassen und Klassen, Arbeitsbesitzern und Arbeitslosen. Prekäre Lebenslage und Selbstentwertung wirken unheilvoll zusammen. Nun ließe sich folgern: je krisenhafter die Zeiten, umso wichtiger die Soziale Arbeit. Aber gewiss ist sie bisher eher Agent(ur) als Agens der Sozialpolitik. Insofern ist der Werbetext zum 7. Bundeskongress Soziale Arbeit 2009 kühn: "Soziale Arbeit übernimmt Verantwortung dafür, soziale Sicherheit zu garantieren. « Ist damit nicht sogar der Staat überfordert? Eine Profession, die der »sozialen Gerechtigkeit « als Leitidee verpflichtet ist, kann sich dieser Verantwortung aber auch nicht entziehen: Sie muss die Sozialpolitik fordern und för- 
dern und - über die materielle Daseinsvorsorge hinaus - zu den »sozialen Grundlagen der Selbstachtung " (John Rawls) und generell zur »Lebensbewältigung unter prekären Bedingungen « beitragen, mikrosozial im Nahraum (u. a. Beziehungsarbeit), mesosozial in Organisationen (u. a. Sozialmanagement), makrosozial in der gesellschaftlichen Öffentlichkeit (u. a. Einstellungswandel).

\section{Schwierigkeiten der Sozialpolitik}

Wenn der Blick auf das Elend hinter dem Fächer folgenlos bliebe, wäre es Voyeurismus. Die zweite Aufgabe besteht deshalb in der Suche nach Lösungen: Strukturell ist die Sozialpolitik, personell - als Dienstleistung - die Soziale Arbeit zuständig. Was funktional so klar zu sein scheint, ist in Wahrheit komplizierter: Das Verhältnis von Sozialarbeit und Sozialpolitik ist vielgestaltig und spannungsreich - zumal in Zeiten, da der Sozialstaat neu buchstabiert wird, in Umbrüchen, die uns alle beunruhigen und viele existenziell bedrohen: Die strukturelle Krise der Arbeit, der Bildung, der Demografie, der Familie, der Wohlstandsverteilung - um nur die wichtigsten zu nennen - und all das auf dem Hintergrund ökonomischer Turbulenzen, die die Betroffenheit aller Bürger schlagartig klarmachen, sind wir doch alle Experten im Geben und Nehmen sozialer Leistungen, pikanter Weise nun auch Manager und »Global Player «. Ob die Krise des Kapitalismus eine Renaissance des Sozialstaats oder seine weitere Schwächung bewirkt, ist noch keineswegs ausgemacht (Marx 2008), und auch nicht, welche Rolle die Soziale Arbeit dabei übernimmt (Bütow/Chassé/ Hirt 2007) - nutznießend, leidtragend, mitgestaltend? Drei Probleme der Sozialpolitik seien beispielhaft herausgegriffen: - Die anonyme sozialstaatliche Hilfe ist Stärke und Schwäche zugleich: Ein Sozialbudget von 700 Milliarden Euro, dessen Verteilungswirkung unklar ist; Leitprinzipien (Gerechtigkeit, Solidarität, Subsidiarität, Nachhaltigkeit, vgl. Sozialwort der Kirchen EKD 1997) die formal anerkannt, aber auslegungsbedürftig sind - womit sich Fragen nach der Definitionsmacht und dem Einfluss der Sozialarbeit stellen.
- Eine Sozialpolitik, die auf Daseinsvorsorge und Geldleistungen reduziert wird, führt zur Ökonomisierung, Bürokratisierung und Verrechtlichung, die Hans Achinger schon vor 50 Jahren beklagte. Dabei verschwimmt der Einzelfall nicht selten in einem Bermudadreieck der Sachzwänge, statt die Effizienz der Leistung und die Selbstverantwortung zu stärken, was vor allem der Sozialen Arbeit aufgetragen ist.

- Zur Steigerung von Sozialleistungen gibt es nur drei Freiheitsgrade: reales Wirtschaftswachstum, Senkung der Investitionsrate, Konsum-Umschichtung. Bisher wählte noch jede Regierung den am wenigsten schmerzhaften Weg des Wirtschaftswachstums. Ausnahme: "Solidarpakt 1991 « und die damaligen Widerstände lassen ahnen, was geschieht, wenn das Wachstum versiegt. Hier lautet die grundsätzliche Frage: "Was schulden wir einander? « (di Fabio/Oermann 2008) und: "Was kann die Soziale Arbeit im Verteilungskampf bewirken? «

\section{Fallstricke des Sozialstaats}

Tragischerweise sind die Mittel des Sozialstaats nicht nur begrenzt, sondern in ihrer Wirkung manchmal auch kontraproduktiv. »Ich bin Profi-Helfer, gehöre zu den helfenden Berufen - also bin ich korrupt «, so beginnt Klaus Dörner $(2008,7)$ sein jüngstes Buch über »Helfende Berufe im Markt-Doping «. Oft würden Gesetze nicht das Helfen verbessern, sondern nur die Leistungsmenge steigern, wobei »das Geld nach dem sinverse care law <... nicht mehr zu den Hilfebedürftigsten, sondern eher zu den profitableren Gesünderen « fließe (ebd. 8). $\mathrm{Zu}$ dieser »Gesundheitsfalle « trügen Denkfehler der Profi-Helfer bei, etwa die Annahme, sie könnten Gesundheit herstellen.

Die Analogie zur Sozialen Arbeit liegt nahe und wird vom Autor auch mehrfach betont. Im Unterschied zum Medizinsystem versteht sie sich aber als Hilfe zur Selbsthilfe, will Befähigungshilfe leisten und Ressourcen aktivieren statt die Selbstsorge zu entsorgen und Gesundes in

\section{»Der Einzelfall verschwindet nicht selten im Bermudadreieck der Sachzwänge«}

Der Staat kann direkt nur über Gesetz und Geld steuern (Niklas Luhmann). Kein Gesetz der Welt aber kann Gesundheit, Freundschaft oder gelingendes Leben verbürgen, auch die Sozialarbeit nicht, wohl aber förderliche Bedingungen schaffen, zu denen mehr gehört als das nackte Überleben. Der Mensch lebt nicht vom Brot allein, auch nicht von Transferzahlungen und Sozialdiensten allein. Von Adam Smith, dem Mitbegründer der Volkswirtschaftslehre, ist der Satz überliefert, Nationalökonomie müsse nicht nur das Realeinkommen sichern, sondern auch die Freiheit, sich ohne Scham in der Öffentlichkeit zu zeigen (Drilling 2004, 49). Ökonomie, Sozialpolitik und Soziale Arbeit stehen sich also in vieler Hinsicht näher, als gemeinhin angenommen wird.
Krankes umzudefinieren. Anders gesagt: Sozialarbeiterinnen und Sozialarbeiter wissen, dass Klienten Experten eigener Art sind und dass Problemlösungen meist nur in Koproduktion gelingen.

Dennoch können kontraproduktive Effekte - beispielsweise durch Förderung von Bequemlichkeit, Trittbrettfahren und »moral hazard « - ebenso wenig ignoriert werden, wie »die moralische Verarmung der Bürger, denen man das Helfen wegnahm « (Dörner 2008, 7). Dieser Funktionsverlust der Haushalte und Nachbarschaften könnte den Sozialstaat am eigenen Erfolg scheitern lassen - wie König Midas, der alles was er berührte, in Gold verwandelte und so verhungern musste.

Erfolge des Sozialstaats? Beispiel Pflegeheim: 75 Prozent der Bürger äußern Angst vor dem Heim, 13 Prozent wollen 
sich lieber vorher umbringen; Beispiel Sonderpädagogik: Förderschulen erzeugen mehr Ärger als Zustimmung; Beispiel Kindertagesstätten: Sie dienen eher dem ökonomischen Verwertungsinteresse als dem Wohl der Kinder. Gesundheitsfalle, Sozialstaatsfalle, Bildungsfalle? - Vielleicht sind es nur mentale Fallen, aber wir sollten uns der Fallstricke ebenso bewusst sein, wie der sozialen Gradienten von Bildung und Gesundheit, um gegensteuern zu können. Das wäre die dritte Aufgabe.

Auf dem Bildungsgipfel vor einigen Monaten stimmten die Akteure in einem Punkt überein: Schulsozialarbeit tut Not! Wenn jede Schule nur eine Stelle schafft, würden 40.000 Schulsozialarbeiterinnen und Schulsozialarbeiter gesucht. Schön für die Profession, aber müsste nicht vorher das herrschende Leitbild und Selektionsprinzip in Frage gestellt werden? »Jeden morgen wacht in Afrika eine Gazelle auf. Sie weiß, sie muss schneller laufen als der schnellste Löwe, um nicht gefressen zu werden. Jeden morgen wacht in Afrika ein Löwe auf. Er weiß, er muss schneller sein als die langsamste Gazelle, wenn er nicht verhungern will. Fazit: Es

ist egal, ob Du Löwe oder Gazelle bist. Wenn die Sonne aufgeht, musst du rennen. " (Geissler in: sozialmagazin 2/2000, 34)

Dieses Bild mag Managern gefallen, kaum der Sozialarbeit. Hieße es doch, sie auf die Rettung Fußkranker zu verpflichten - damit andere umkommen. Die Postmoderne verspricht mehr Autonomie, blockiert aber für viele den Zugang dorthin, das Freiheitsverlangen schlägt sogar um in neue Abhängigkeiten - von Erfolg, Fitness, Konsum (Mühlum 2007, 79). Die wachsende Zahl der Verliererschicksale - nicht fit, nicht jung, nicht clever lässt sich jedoch nur zeitweise verdrängen oder mit wachsenden Kosten ausgrenzen, ohne das Leiden an der Gesellschaft (Bourdieu 1997) und an der Sinnlosigkeit (Frankl 1978) zu mindern.

Die aktuelle Krise stellt daher nicht nur unser Wirtschaftsmodell, sondern auch das Gesellschaftsmodell in Frage. Soziale Arbeit könnte - über Sozialpolitik hinaus - zu einem neuen Lebensstil ermutigen, vorausgesetzt, sie ist selbst dazu bereit. Dazu gehört auch, sich von der Selbstblockade des »doppelten Man-

\section{Zusammenarbeit von Sozialpolitik und Sozialarbeit nötig}

\section{Deutsche GesellsChaft FüR Soziale Arbeit}

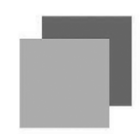

Forum für Wissenschaft und Praxis und Hochschullehrer soziale Probleme so hautnah erleben, erforschen und reflektieren wie kaum eine andere Berufsgruppe, fordern sie, in der Sozialpolitik stärker Gehör zu finden. Dem soll u. a. eine Grundsatzerklärung dienen, die in nächster Zeit diskutiert und beschlossen werden soll. Mit dieser »Jenaer Erklärung « soll die Öffentlichkeit auf die Notwendigkeit einer Politikgestaltung hingewiesen werden, die den Ansprüchen der Bürgerinnen und Bürger an den Sozialstaat und die soziale Sicherung gerecht wird. Bei den Diskussionen in Jena wurde deutlich, dass Sozialpolitik und Sozialarbeit erst am Beginn der Zusammenarbeit stehen, die im Interesse der sozial Schwächeren dieser Gesellschaft intensiviert werden muss. Auf der Website der Deutschen Gesellschaft für Soziale Arbeit können der Tagungsbericht von Prof. Dr. Albert Mühlum sowie die aktuelle Fassung der »Jenaer Erklärung« als PDF-Dokumente kostenfrei heruntergeladen werden. Ebenso stellen einige Referenten ihre Vorträge aus Jena hier zur Verfügung (www.www.deutsche-gesellschaftfuer-soziale-arbeit.de, Rubrik Mitteilungen, Online-Mitteilung 133).

Deutsche Gesellschaft für Soziale Arbeit e. V., Postfach 11 29, 74370 Sersheim, Telefon 07042 3948, Fax 07042 815540, E-Mail info@dgsinfo.de, Internet http://www.deutschegesellschaft-fuer-soziale-arbeit.de dats « zu befreien, also neben Trägerauftrag und Klientenauftrag den Professionsauftrag (= Selbstverpflichtung der Profession) als dritte Bezugsgröße ernst zu nehmen (Silvia Staub-Bernasconi: Triple-Mandat).

Im übrigen kann Armut nicht auf Einkommensarmut, Sozialstaat nicht auf Umverteilungsstaat reduziert werden. Für Amartya Sen sind weder Nutzen (im Sinne des Utilitarismus) noch Grundgüter (im Sinne John Rawls), sondern Grundrechte, Freiheit und Fähigkeit zentral, »ein mit Gründen schätzenswertes Leben zu wählen «(Sen 2000, 94). In seinem »Fähigkeitsansatz « sind die Fähigkeiten zu handeln (»capabilities «), tatsächlichen Möglichkeiten (»functionings «) und erreichten Ziele (»achievements «) zu unterscheiden (Drilling 2004, 45ff). Armut wäre dann ein Mangel an Verwirklichungschancen, der oft mit der Einkommenssituation eines Haushalts zusammenhängt, aber weit darüber hinaus geht.

Positiv formuliert: Lebensqualität hat viele Facetten, denen das LebenslageKonzept der Sozialpolitik und das Lebenswelt-Konzept der Sozialen Arbeit am ehesten entsprechen. Ob diese Konzepte der Politik und der Öffentlichkeit bekannt sind, ist allerdings ebenso fraglich, wie deren Wissen um die Bedeutung der Sozialen Arbeit für die Bildung von $\mathrm{Hu}-$ man- und Sozialkapital.

\section{Spiritualität als Ressource}

Das Glück der Menschen ist nicht Aufgabe der Politik. Allzu leicht würde daraus ein Beglückungszwang, wie die Geschichte zeigt. Der Staat hat mit Leidvermeidung (Karl Popper) genug zu tun. Aber gilt das auch für die Soziale Arbeit? Zu ihrem unverwechselbaren Kern gehören das Konzept »Person-in-der-Situation " und eine personale Beziehung, die sich berufsethisch und methodisch im »Verstehen und Achten « (Mührel 2005) ausdrückt. Menschen ganzheitlich zu sehen heißt auch, ihr Streben nach Glück und die Suche nach Sinn zu würdigen, dennoch kommt die Kategorie Glück in der beruflichen Sozialarbeit kaum und in ihren Theorien überhaupt nicht vor, was im wesentlichen auch für das Thema Spiritualität gilt (dazu und zum Folgenden: Mühlum 2007, 82 ff.): 
»Glücklich ist, wer alles hat, was er will«, meinte der Heilige Augustinus, der die zahlreichen Glücksdefinitionen seiner Zeit wohl kannte. Trivial oder genial? Der Satz zwingt zum Weiterfragen: Was will ich im letzten und was kann ich verlässlich haben? Philosophie beginnt, wenn darüber nachgedacht wird. Erich Fromms »Haben oder Sein « (2005) ist ein solcher Versuch. Auch Elisabeth Lukas (2003) geht mit der Logotherapie davon aus, dass wir trotz eines Überschusses an Haben an einem Defizit des Seins leiden, das im Bezug auf ein Sollen inhaltlich anzureichern wäre, wenn das Leben glücken soll.

»Was willst Du? «Schon die Frage löst in Therapiegruppen heftige Gefühle aus. Nach Irvin Yalom entsteht in Minuten eine emotionsgeladene Atmosphäre. Männer und Frauen werden im Innersten aufgewühlt. Sie rufen nach den Menschen, die sie verloren haben - verstorbene oder entschwundene Eltern, Partner, Kinder, Freunde. »Ich will dich wiedersehen ", »Ich will, dass du stolz auf mich bist «, »Ich will die Kindheit erleben, die ich nie hatte «, »Ich will geliebt werden, will meinem Leben einen Sinn geben, will in Erinnerung bleiben " (Yalom 1999, 9 f.). So viele Sehnsüchte und Schmerzen. Sie erinnern daran, dass die tiefsten Wünsche unerfüllt - vielleicht unerfüllbar sind. "Glücklich ist, wer alles hat, was er will.« Was auf den ersten Blick wie eine "Anleitung zum Unglücklichsein « (Watzlawick) klingt, birgt eine spirituelle Weisheit: Wir können unser Wollen ändern, das ist mit Umkehr im biblischen Sinne gemeint und sollte bei jedem lösungsorientierten Vorgehen mit bedacht werden (vgl. auch das Reframing in Therapie und klinischer Sozialarbeit).

Aber was, wenn die Demenz des Vaters, der Verlust des Partners, der Tod eines Kindes beklagt wird. Was ist dann noch wichtig - oder gerade dann? Wenn die Frage nach dem Sinn systemimmanent nicht zu beantworten ist - und wie könnte das sein am Grab eines geliebten Menschen -, bleibt nur, das System zu übersteigen, beispielsweise im Glauben, dass der Tod nicht das letzte Wort hat. Dieses Transzendieren fiel aber einer Wissenschaftsgläubigkeit zum Opfer, die selbst irrationale Züge trägt und die Sinnfrage verfehlt. Ein Gefühl der Sinnhaftigkeit aber ist im »Sense of Coherence«Konzept der Salutogenese das wichtigste
Kriterium subjektiver Gesundheit. Im Umkehrschluss macht es die verheerende Wirkung des Demoralisierungssyndroms sozial benachteiligter Menschen umso deutlicher.

Da die Soziale Arbeit unvermeidlich mit Verlust - Verlustangst, Verlusterfahrung - konfrontiert ist, muss sie, über »sozialpolitische Bedürfnisse « hinaus, sensibel für die Sinnfrage und spirituelle Anliegen sein. »Denn jede Rede von Ganzheitlichkeit ist absurd, wenn sie die Frage nach den >letzten Dingen und die Antwort(ver)suche der Beteiligten ausklammert.«(Mühlum

\section{Literatur}

Achinger, H. (1958): Sozialpolitik als Gesellschaftspolitik. Frankfurt am Main.

Bourdieu, P. et al. (1997): Das Elend der Welt. Zeugnisse und Diagnosen alltäglichen Leidens an der Gesellschaft. Konstanz.

Bütow, B./Chassé, K. A./Hirt, R. (Hg.) (2007): Soziale Arbeit nach dem Sozialpädagogischen Jahrhundert. Positionsbestimmungen Sozialer Arbeit im Post-Wohlfahrtsstaat.

DBSH (1997): Berufsethische Prinzipien. Essen.

Di Fabio, U./Oermann, N. O. (Hg.) (2008): Was schulden wir einander? Berlin.

Dörner, K. (2008): Helfende Berufe im Markt-Doping. Wie sich Bürger- und ProfiHelfer nur gemeinsam aus der Gesundheitsfalle befreien. Neumünster.

Drilling, M. (2004): Young urban poor. Abstiegsprozesse in den Zentren der Sozialstaaten. Wiesbaden.

EKD/Katholische Bischofskonferenz (Hg.) (1997): Zur wirtschaftlichen und sozialen Lage in Deutschland. Bonn/Hannover.

Frankl, V. E. (1978): Das Leiden am sinnlosen Leben. 7. Aufl. Freiburg im Breisgau. Fromm, E. (2005): Haben oder Sein. Die seelischen Grundlagen einer neuen Gesellschaft. Nachdruck, Stuttgart.

Lallinger, M./Rieger, G. (Hg.) (2007): Repolitisierung Sozialer Arbeit. Engagiert und professionell. Stuttgart.

Lewkowicz, M./Lob-Hüdepohl, A. (Hg.) (2003): Spiritualität in der Sozialen Arbeit. Freiburg im Breisgau.

Lob-Hüdepoh, A./Lesch, W. (Hg.) (2007): Ethik sozialer Arbeit. Ein Handbuch. Paderborn.

Lukas, E. (2003): Spannendes Leben: In der Spannung zwischen Sein und Sollen ein Logotherapiebuch. 3. Aufl. München.

Marx, R. (2008): Das Kapital. München.

Maus, F./Nodes, W./Röh, D. (2008): Schlüsselkompetenzen der Sozialen Arbeit für die Tätigkeitsfelder Sozialarbeit und Sozialpädagogik. Schwalbach.

Mühlum, A. (2006): Spiritualität - (k)ein Thema der Sozialen Arbeit? In: Forum Sozial 1/2006, 9-12.

Mühlum, A. (2007): Spiritualität - eine vergessene Ressource der Sozialen Arbeit. In: Homfeldt, H. G. (Hg.): Soziale Arbeit im Aufschwung zu neuen Möglichkeiten. Baltmannsweiler, 78-90.

Mühlum, A./Olschowy, G./Oppl, H./Wendt, W. R. (1986): Umwelt - Lebenswelt. Beiträge zu Theorie und Praxis ökosozialer Arbeit. Frankfurt am Main.

Mührel, E. (2005): Verstehen und Achten. Philosophische Reflexionen zur professionellen Haltung in der Sozialen Arbeit. Essen.

Sen, Amartya (2000): Ökonomie für den Menschen. Wege zur Gerechtigkeit und Solidarität in der Marktwirtschaft. München.

Staub-Bernasconi, S. (2007): Soziale Arbeit: Dienstleistung oder Menschenrechtsprofession? In: Lob-Hüdepoh/Lesch (Hg.), Paderborn, 20-53.

Wendt, W. R. (2008): Geschichte der Sozialen Arbeit. 5. Aufl. 2 Bände., Stuttgart. Yalom, Irvin (1999): Die Liebe und ihr Henker \& andere Geschichten aus der Psychotherapie. München. 
2007, 78). Das ist auch ein Plädoyer für eine Sozialarbeitsphilosophie, die über berufsethische Prinzipien (DBSH 1997) und Schlüsselkompetenzen (Maus/Nodes/Röh 2008) hinausgeht und Spiritualität als Ressource (wieder) entdeckt (vgl. Lewkowicz/Lob-Hüdepohl 2003; Lob-Hüdepohl/Lesch 2007).

"Den Menschen auf seine biologische Substanz, genetische Ausstattung, biochemische oder soziale Funktionen zu reduzieren, wäre ein dürftiges Bild vom Menschen. Was sollte dann daran hindern, auszumerzen, was nicht gefällt? Kinder zu dressieren, statt zu bilden; Bürger zu manipulieren, statt zu überzeugen; Leidende umzubringen, statt ihnen die Hand zu halten? Dem Diktat der Ökonomie und der Biotechnologie der Life Sciences würde am Ende die Sozialtechnologie der Social Sciences zur Seite treten - assistiert von der Sozialen Arbeit. « (Mühlum 2006, 11)

Wer dies als Horrorszenario empfindet, muss eine Vorstellung von der genuinen Würde des Menschen haben, die nicht zur Disposition steht - was pointiert im Konzept der Menschenrechtsprofession (Staub-Bernasconi 2007) Ausdruck findet. Im übrigen muss sich jeder im Hilfeprozess selbst fragen: Nach den Grundfiguren seines Lebens, den Vorstellungen von Gott und Welt, Mensch und (Un-) Endlichkeit. Vor einer zu schnellen Antwort sei mit Stanislaw Jercy Lec gewarnt: »Ob ich Atheist bin, das weiß Gott allein. «Wie die Antworten auch ausfallen mögen, in den Krisen und Grenzsituationen des Lebens stellen sich die Fragen stets neu - und je älter man wird, umso drängender (Müh$\operatorname{lum} 2007,88)$.

\section{Fazit}

Soziale Arbeit als Agens der Politik? Ja und Nein. Nein, wenn damit ein politisches Mandat ohne demokratische Legitimation gemeint ist. Ja, wegen des ureigenen Auftrags der Profession, zu förderlichen Lebensbedingungen, sozialer Kultur und Verwirklichungschancen beizutragen. Dazu gilt es

- erstens den »Blick hinter den Fächer « zu werfen, um Probleme verstehen und adäquate Lösungen entwickeln zu können (siehe Sozialarbeitsforschung)

- zweitens auf strukturelle Abhilfe zu drängen, also auch Politiker zu bedrän- gen, die Voraussetzungen für eine menschenwürdige Existenz zu schaffen (siehe geplante "Jenaer Erklärung " der Deutschen Gesellschaft für Soziale Arbeit)

- drittens die Fähigkeiten der eigenen Zunft zu fördern, klientbezogen ebenso effektiv und effizient zu handeln wie in Institutionen (siehe Professionalität)

- viertens eine Zivilgesellschaft zu schaffen, die den sozialen Raum kultiviert und Bürger motiviert, füreinander Ver- antwortung zu übernehmen (siehe bürgerschaftliches Engagement).

Nur ein solcher Politikmix wird den künftigen Gesamthilfebedarf abdecken können - auf dem Weg zu einer sozial integrierten Gesellschaft. Aber, um mit einem Aphorismus von Vaclav Havel zu schließen: Hoffnung ist nicht die Überzeugung, dass etwas gut ausgeht, sondern die Gewissheit, dass etwas (hier: das sozialberufliche Bemühen) Sinn hat, ohne Rücksicht darauf, wie es ausgeht.

\section{Die Verfahrensregeln in der Anwendung}

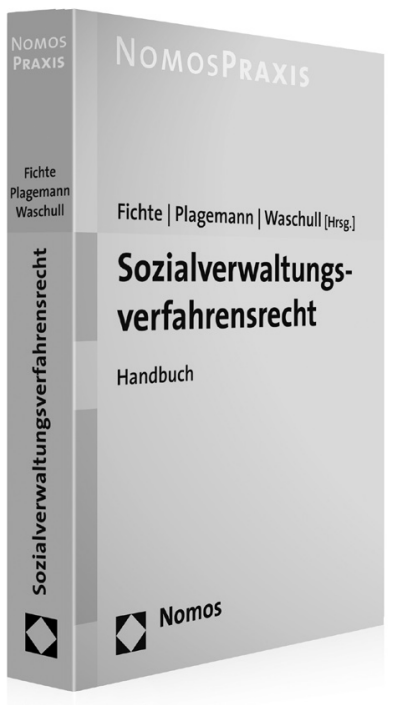

\author{
Sozialverwaltungs- \\ verfahrensrecht \\ Handbuch \\ Herausgegeben von RiBSG Dr. Wolf- \\ gang Fichte, RA Prof. Dr. Hermann \\ Plagemann, FAMedR und FASozR, und \\ RiLSG Prof. Dr. Dirk Waschull \\ 2008, 433 S., brosch., 49,- $€$, \\ ISBN 978-3-8329-2610-6
}

Das Handbuch berücksichtigt die verschiedenen Herangehensweisen der Sozialbehörden, Sozialgerichte und der Anwaltschaft. Anhand typischer Anwendungsprobleme stellen die Autoren die Verbindung von Verfahrensrecht und materiellem Recht her. Die Unterschiede zwischen dem Sozialverwaltungsverfahren nach SGB X und dem VwVfG-Verfahren werden verdeutlicht. Zahlreiche Beispiele, Antragsmuster, Gebührenhinweise sowie Ausführungen zum (einstweiligen) Rechtsschutz erhöhen den praktischen Nutzen.

Aus dem Inhalt:

- Verwaltungsverfahren

- Rechtskonkretisierung und -gestaltung durch Verwaltungsakt

- Rücknahme und Widerruf von Verwaltungsakten

- Vertragsrecht

- Sozialdatenschutz

- Zusammenarbeit und Erstattungsansprüche der Leistungsträger untereinander und gegenüber Dritten

Bitte bestellen Sie im Buchhandel oder versandkostenfrei unter $>$ www.nomos-shop.de 